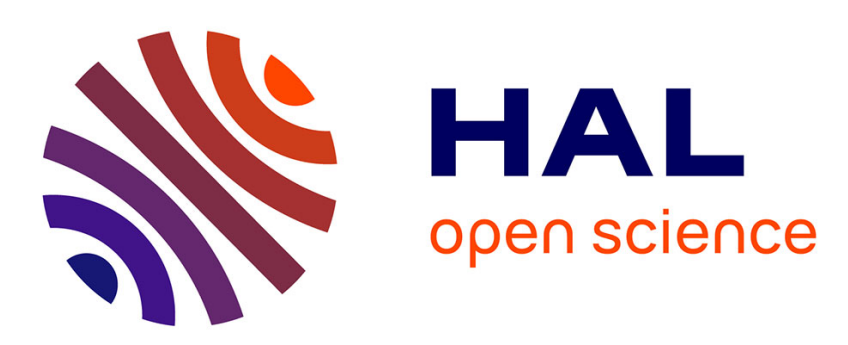

\title{
A General approach to trace-checking in distributed computing systems
}

Claude Jard, Thierry Jéron, Guy-Vincent Jourdan, Jean-Xavier Rampon

\section{To cite this version:}

Claude Jard, Thierry Jéron, Guy-Vincent Jourdan, Jean-Xavier Rampon. A General approach to trace-checking in distributed computing systems. [Research Report] RR-2238, INRIA. 1994. inria00074432

\section{HAL Id: inria-00074432 \\ https://hal.inria.fr/inria-00074432}

Submitted on 24 May 2006

HAL is a multi-disciplinary open access archive for the deposit and dissemination of scientific research documents, whether they are published or not. The documents may come from teaching and research institutions in France or abroad, or from public or private research centers.
L'archive ouverte pluridisciplinaire HAL, est destinée au dépôt et à la diffusion de documents scientifiques de niveau recherche, publiés ou non, émanant des établissements d'enseignement et de recherche français ou étrangers, des laboratoires publics ou privés. 


\section{A General Approach to Trace-Checking in Distributed Computing Systems}

Claude Jard, Thierry Jéron, Guy-Vincent Jourdan et Jean-Xavier Rampon

$\mathbf{N}^{\circ} 2238$

Mars 94

PROGRAMME 1

Architectures parallèles,

bases de données,

réseaux et systèmes distribués

\section{apport}

derecherche 



\title{
RINRIA
}

\section{A General Approach to Trace-Checking in Distributed Computing Systems}

\author{
Claude Jard, Thierry Jéron, Guy-Vincent Jourdan et Jean-Xavier Rampon \\ Programme 1 - Architectures parallèles, bases de données, réseaux et systèmes distribués \\ Projet Pampa
}

Rapport de recherche ${ }^{\circ} 2238$ - Mars $94-18$ pages

\begin{abstract}
The problem of checking the correctness of distributed computations arises when debugging distributed algorithms, and more generally when testing protocols or distributed applications. For that purpose, one describes the expected behavior (or suspected errors) by a global property: for example, a predicate on process variables, or the set of admissible orderings on observable events. The problem is to check whether this property is satisfied or not during the execution. A relevant model for this study is the partial order of message causality and the associated state graph, called "lattice of consistent cuts". In this paper, we propose a general approach to trace checking, based on partial order theory.
\end{abstract}

Key-words: Distributed computation, debugging parallel programs, predicate checking, timestamps, causality order, lattice of consistent cuts

(Résumé : tsvp)

A paraitre dans " $14^{\text {th }}$ International Conference on Distributed Computing Systems", Poznan, Pologne, Juin 1994

Unité de recherche INRIA Rennes

IRISA, Campus universitaire de Beaulieu, 35042 RENNES Cedex (France)

Téléphone : (33) 99847100 - Télécopie : (33) 99847171 


\section{Une approche générale pour la vérification de traces dans les systèmes répartis}

Résumé : Le problème de la vérification de la correction de calculs répartis survient lorsque l'on souhaite déboguer des algorithmes distribués, et plus généralement lorsque l'on teste des protocoles ou des applications réparties. Dans ce but on décrit le comportement attendu (ou les erreurs supposées) par une propriété globale: par exemple un prédicat portant sur les variables de processus, ou l'ensemble des ordres admissibles entre événements observables. Le problème est de vérifier si cette propriété est satisfaite ou non au cours de l'exécution. Un modèle adéquat pour cette étude est celui de l'ordre partiel de causalité et le graphe des états associés appelé "treillis des coupes cohérentes". Dans cet article, nous proposons une approche générale à la vérification de trace fondée sur la théorie des ordres partiels.

Mots-clé : Calcul réparti, débogage de programmes répartis, vérification de prédicats, estampillage, ordre de causalité, treillis des coupes cohérentes 

system. Any pronouncement on the correctness of a particular test must be valid for any possible interleaving of events compatible with the causal ordering induced by the observed computation.

\subsection{Work position}

In the last ten years, there were several propositions to help in distributed debugging, even in the special case we called trace-checking or predicate detection. The pioneering work in that area was presented in [CL85], and gives an algorithm to detect stable predicates, which uses global snapshots. Since then, the scientific community tries to extend this work to larger classes of properties, said "unstable" [GW92, CM91, BR94]. Different algorithms were proposed to deal with local predicates, conjunction and disjunction of local predicates, sequencing of local predicates... All these algorithms suffer from a lack of homogeneous theoretical structure. Consequently, the different proofs of the algorithms do not even help since they appear ad-hoc. Faced to this situation, we were tempted to study in depth the structure of the lattice of global states (or consistent cuts, or ideals). We think this lattice is a good candidate to found the reflection on predicate detection. The paper defends such a position. We show that understanding the dynamic structure of the lattice directly leads to general algorithms which detect larger classes of predicates than in the previous literature.

The remainder of the paper is organized as follows. We start by exposing the problem of trace observation. We lay stress on the notion of observable event, and on the vector coding of subsets of events. We then introduce a classification of properties based on the ability to decide them on-line. Finally, we show how to check properties on traces. These properties are expressed by finite automata. Though the techniques are well-known in the area of modelchecking, we present an algorithm which strictly extends the class of properties that were presented in previous works in distributed debugging.

\section{Trace observation}

This section is devoted to the problem of trace observation in a distributed environment. We start by defining some terminology which stems from the theory of partial orderings and lattices. Then we recall the notions of "causal relation" and "consistent cuts" for observable events. The remainder of the section is devoted to the on-line reconstruction of the causal relation by an observer.

\subsection{Terminology}

A partially ordered set ( poset) $\widetilde{P}=\left(P, \leq_{\widetilde{P}}\right)$ is a set $P$ of elements, together with an antisymmetric, reflexive and transitive binary relation $\leq_{\widetilde{P}}$. In addition, if $x \leq_{\widetilde{P}} y$ and $x \neq y$, then we write $x<_{\widetilde{P}} y$. If $x \leq_{\widetilde{P}} y$ or $y \leq_{\widetilde{P}} x$ we say that $x$ and $y$ are comparable in $\widetilde{P}$. Otherwise, $x$ and $y$ are said incomparable in $\widetilde{P}$. If $x \leq_{\widetilde{P}} y$, then $x$ is a predecessor of $y$ in $P$ and $y$ is 
a successor of $x$ in $P$. We say that $y$ is an immediate successor of $x$ (or $x$ is an immediate predecessor of $y$ ) or $y$ covers $x$ and note $x<_{\widetilde{P}} y$ if $x<y$ and $\forall z \in P, x<_{\widetilde{P}} z \leq_{\widetilde{P}} y \Rightarrow z=y$. The directed graph associated to this covering relation is denoted $\operatorname{Cov}(P)=\left(\stackrel{P}{P}, E_{P}\right)$.

Let $A$ be a subset of $P$, the suborder of $\widetilde{P}$ on $A$ is $\widetilde{P / A}=\left(A, \leq_{\widetilde{P}}\right)$. An antichain (resp. a chain) is a subset of $P$ in which every pair of elements is incomparable (resp. comparable). A chain decomposition in $k$ chains of $\widetilde{P}$ is a partition $\left\{P_{i}\right\}_{i \in[1 \ldots k]}$ of $P$ in which every $P_{i}$ is a chain of $\widetilde{P} . \operatorname{Max}(A, \widetilde{P})=\left\{a \in A, \forall x \in A, \neg\left(a<_{\widetilde{P}} x\right)\right\}$ is the set of maximal elements of $A$ in $\widetilde{P}$. Similarly, $\operatorname{Min}(A, \widetilde{P})=\left\{a \in A, \forall x \in A, \neg\left(x<_{\widetilde{P}} a\right)\right\}$ is the set of minimal elements. $A$ has an infimum (resp. a supremum) in $\widetilde{P}$ if $\left|\operatorname{Max}\left(\left\{x \in P, \forall y \in A, x \leq_{\widetilde{P}} y\right\}, \widetilde{P}\right)\right|=1$ (resp. $\left.\left|\operatorname{Min}\left(\left\{x \in P, \forall y \in A, y \leq_{\widetilde{P}} x\right\}, \widetilde{P}\right)\right|=1\right) . \widetilde{P}$ is a lattice iff every pair of elements has a supremum and an infimum in $\widetilde{P}$.

Let $x \in P, \downarrow_{P} x=\left\{y \in P, y \leq_{\widetilde{P}} x\right\}$ (resp. $\uparrow_{P} x=\left\{y \in P, x \leq_{\widetilde{P}} y\right\}$ ) is the predecessor set (resp. the successor set) of $x$ in $\widetilde{P} \cdot \downarrow_{P}^{i m} x=\left\{y \in P, y \prec_{\widetilde{P}} x\right\}$ (resp. $\uparrow_{P}^{i m} x=\left\{y \in P, x \prec_{\widetilde{P}} y\right\}$ ) is the immediate predecessor set (resp. the immediate successor set) of $x$ in $\widetilde{P}$.

A linear extension of a poset $\widetilde{P}$ is a maximal chain $C$ onto $P$ which preserves $\leq_{\widetilde{P}}$, i.e.: $x \leq_{\widetilde{P}} y \Rightarrow x \leq_{\widetilde{C}} y$.

An ideal $A$ of a poset $\widetilde{P}$ is a subset closed by precedence: $\forall x \in A, \forall y \in P, y \leq_{\widetilde{P}} x \Rightarrow$ $y \in A$. When $\operatorname{Max}(A, \widetilde{P})$ is reduced to a singleton $\{x\}$, we say that $A$ is a sup-irreducible associated to $x$. Observe that the set of maximal elements in an ideal forms an antichain and that this provides a one-to-one corresponding between the ideals and the antichains of a poset. The set of ideals $I(P)$ of a poset $\widetilde{P}$, ordered by set inclusion, forms a distributive lattice $\widetilde{I(P)}$ (see $[$ Bir67]).

\subsection{Message causality}

Formally reasoning on execution traces requires a simple mathematical model. Two different levels can be distinguished:

- The user's level, where the programmer defines what he wants to trace (variable changes, calls to sending procedures, ...). These are actions occurring during the computation. The action occurrences are called observable events. The action name defines the event type (or label).

- The run-time level which induces some constraints on the relative ordering of observable events. Precisely, that is the causality due to the physical exchange of messages between processes, ensuring that the sending of a message must always precede its reception.

An example of a trace is given in figure 2(a). The observable events are figured as dots on the "time-line" of processes. Arrows represent the message exchanges.

$\mathrm{RR} n{ }^{\circ} 2238$ 
Let us consider a finite set of processes, denoted by $P_{1}$ to $P_{n}$ (one considers for simplification this set is known in advance and stable). We denote $E=X \uplus \bar{X} \uplus O$ the finite set of events occurring during the execution ( $\uplus$ denotes the disjoint union). $X$ contains all the sending events (unobservable in principle), $\bar{X}$ contains the corresponding receptions (for the sake of simplicity, communication channels are considered point-to-point) and $O$ is the set of observable events. One can consider that $E$ is split up into the disjoint subsets $E_{i}$ of events that are local to $P_{i}: E=\biguplus_{1 \leq i \leq n} E_{i}$.

Let $\prec_{i}$ be the sequential ordering of events appearing on process $P_{i}$. The causal relationship (whose definition is given in [Lam78]) in $E^{2}$ is the smallest relation $\prec$ satisfying :

1. $\forall i \in\{1 . . n\}, \forall x, y \in E_{i}, \quad x \prec i \quad y \Longrightarrow x \prec y$

2. $\forall x \in X, \quad x \prec \bar{x}$

3. $\prec$ is transitive

$\prec$ is a partial order. In the sequel, we will only consider the suborder $\widetilde{O}$ induced by the set $O$ of observable events partially ordered by the relation $\prec$. This abstraction capability is interesting as it allows us to not consider communication events. This leads to our trace model: a distributed trace is modeled by a finite poset $\widetilde{O}$, split up into $n$ disjoint chains and equipped with a labeling function $\lambda$ from $O$ to an action alphabet $\Sigma$. We say that a trace satisfies the NIVI condition (for "non invisible interaction") if on each process, between a receive event and a following send event there exists at least one observable event i.e. $\forall i \in[1 . . n], \forall \bar{y}_{i} \in \bar{X}_{i}, \forall x_{i} \in X_{i}, \bar{y}_{i} \prec_{i} x_{i} \Longrightarrow \exists o_{i} \in O_{i}$ s.t. $\bar{y}_{i} \prec_{i} o_{i} \prec_{i} x_{i}$.

In practice, the observer has to reconstruct the poset $\widetilde{O}$ from the observed events. More precisely, it has to reconstruct the covering digraph of $\widetilde{O}$, which Hasse diagram is shown in figure 2(b). So we need to associate some kind of coding to events in order to make it possible. The observer receives the event $e$ together with the process number in which $e$ occurs and the coding. Moreover, the construction has to be done on-line. But when an event $e$ is received by the observer, there may be some events which belong to the past of $e$ in the computation and which have not been observed yet. For example, in figure 2 assume that all events but events 5 and 8 have already been observed, and suppose that the observer now receives 8 . The event 5 , which is an immediate predecessor of 8 , is missing. There are two possible techniques in that case. The first one is to compute the suborder of the real causal order on the already observed events. In our example, that means that 1 becomes an immediate predecessor of 8 in the suborder. When 5 arrives, the edge between 1 and 8 has to be removed as it becomes a transitive edge. The second possibility is to wait for a linear extension, that is when an event $e$ is observed and there is some missing events in the past of $e$, the observer must wait all missing events before taking $e$ into account. The observer still works on-line as we can expect that the lowest events in the order will not be systematically received after the highest ones. Sections 2.3 and 2.4 present two ways of coding the causal relation and algorithms to extract the covering digraph of the poset from its coding.

Once we have constructed a certain part of the poset $\widetilde{O}$, say the subposet $\widetilde{O^{\prime}}$, we may construct its ideal lattice. If $\widetilde{O^{\prime}}=\widetilde{O}$, then this lattice is the "lattice of consistent cuts" (see 



suborders on ideals of $\widetilde{O}$. So when the observer receives an event $e$, a new suborder can be computed only if all predecessors of $e$ have already been observed. Then we can awake the construction of suborders using events which have only $e$ as missing event in their past. We use lists waitingfor $[v, i]$, which contain observed events which at least wait for the $v^{\text {th }}$ event $z$ on the process $P_{i}$ to get all their past in the set of observed events. Those events may wait for $z$ either because $z$ is missing or because $z$ is also waiting for other events. To each event $y$ we associate a counter missing, which counts the number of index $i$ such that $\exists v, y \in$ waitingfor $[v, i]$. Thus missing is null if all events in the past of $y$ have already been observed. For each event $x$, the algorithm constructs an array $L_{x}$ of observed events such that for $i \neq \operatorname{site}(x), L_{x}[i]$ is the $D_{x}[i]^{\text {th }}$ event in $P_{i}$ and $L_{x}[\operatorname{site}(x)]$ is the $D_{x}[\operatorname{site}(x)]-1^{\text {th }}$ event in $P_{\text {site }(x)}$. The sentence "compute the list of immediate predecessors of $x$ " in procedure Wake-Up can be performed in two steps. Firstly, we associate to each event $x$ a list $\mathcal{L}_{x}$ of events such that $\mathcal{L}_{x}[i]=\max \left\{y \in O_{i}, y \prec x\right\}$ (this list corresponds to the set $L$ in the previous section). This is done in $\mathcal{O}\left(n^{2}\right)$ since $\mathcal{L}_{x}[i]=\max \left\{y \in \bigcup_{y \in L_{x}} \mathcal{L}_{y}[i] \cup L_{x}[i]\right\}$ Note that this also gives us the transitive closure of the poset since this construction can be used to compute the vector timestamps $\Delta(x)$. Secondly, we deduce $\downarrow_{0}^{i m} x$ from $\mathcal{L}_{x}$ in $\mathcal{O}\left(n^{2}\right)$ since $\downarrow_{0}^{i m} x=\left\{y \in \mathcal{L}_{x}[i], \forall z \in \mathcal{L}_{x}, \neg(\Delta(y)[i] \leq \Delta(z)[i])\right\}$. After this, we can proceed with the construction of the lattice or the verification of properties (see section 4). Using the chain decomposition induced by processes of $\widetilde{O}$, the algorithm runs in $\mathcal{O}\left(|O|+n^{2}\right)$ for each event. 


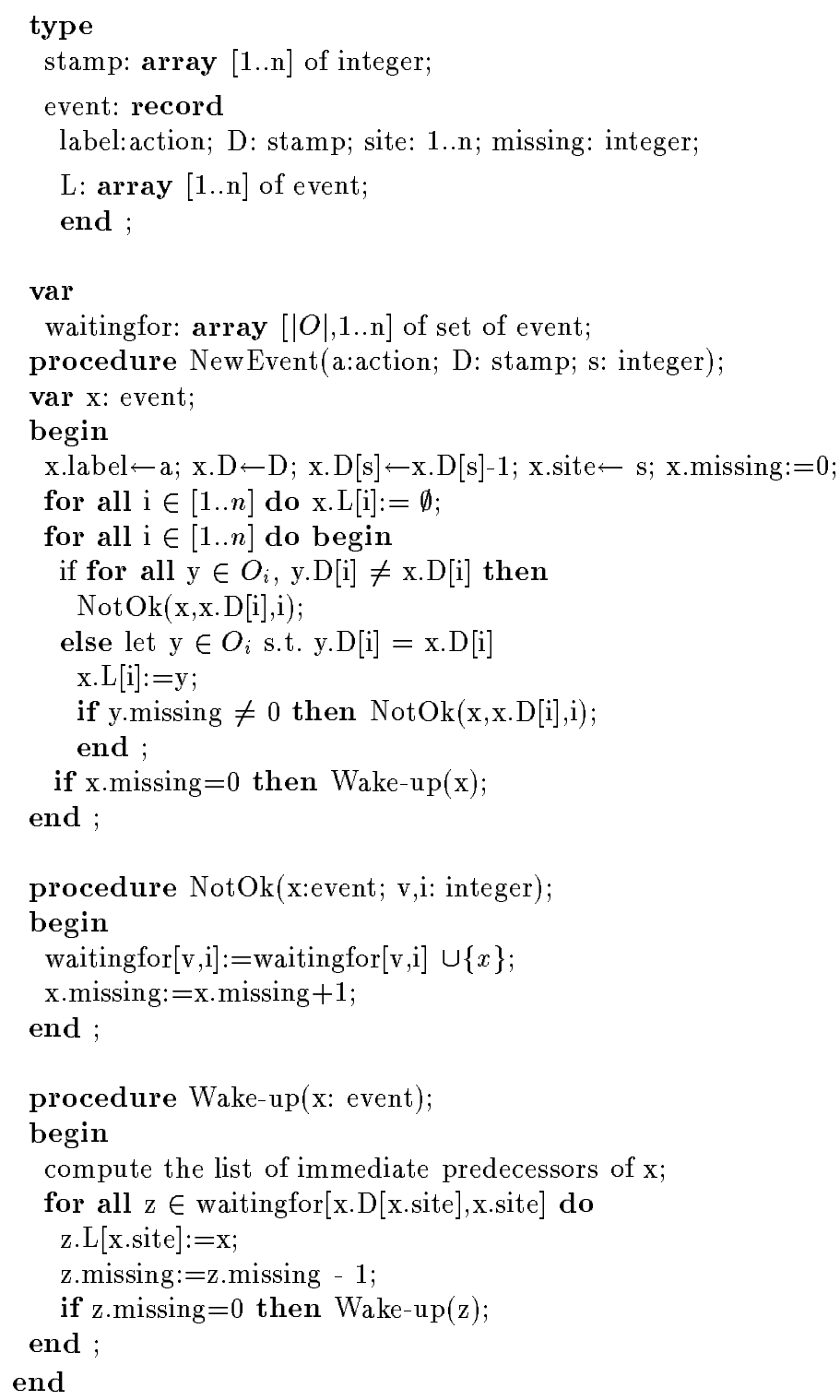

\subsection{Garbage collection}

The size of the ideal lattice is a practical problem. In order to save some memory during its construction and verification, we informally give the clue to remove the garbage part of the lattice. For the sake of simplicity, we assume that the subposet $\widetilde{O^{\prime}}$ is constructed according 
to a linear extension of the complete poset. Let $O_{1}, \ldots O_{n}$ be a chain decomposition of $\widetilde{O^{\prime}}$. And let $V$ be a vector of size $n$ such that $V(i)=y_{i}$ where $y_{i} \in O_{i}$ and $\forall z \in O_{i}, z \leq_{\widetilde{O}^{\prime}} y_{i}$. We assume that each component of $V$ is defined (i.e. we have already observed one event on each site). Let $G=\left\{z \in O^{\prime}, \forall i \in[1 \ldots n], \neg\left(z \geq_{\widetilde{O^{\prime}}} y_{i}\right)\right\} . G$ is an ideal of $\widetilde{O^{\prime}}$. Then, on the ideal lattice of $\widetilde{O^{\prime}}$, an ideal $I$ can be removed iff $I \subseteq G$. This can be easily done using techniques detailed in [DJR93].

\section{The complexity of property checking}

\subsection{What kind of checking?}

Independently to the problem of which kind of property we want to check, we have to consider what kind of checking we want. Two main kinds of checking can be considered. In one hand, we may want to enlight one (generally the first) consistent cut verifying a property. For example, the property is an error detection defined on conjunction of local predicates. This kind of checking can be efficiently performed on the poset since it can be done without backtracking. On the other hand, we may be interested in enumerating all consistent cuts verifying the property. There may be several reasons to do that: counting, computing some average value, or listing every "minimal" snapshot verifying the property (i.e. an antichain in the ideal lattice). In this case, checking on the poset will induce backtracking and may lead to a complexity equivalent (or worse) to the construction and the verification on the ideal lattice. This leads to the definition of a general method to check such properties directly on the lattice, instead of ad-hoc methods. Section 4 is a first step in this direction.

\subsection{A sketch of classification of properties}

Usually, one distinguishes between local properties, which can be decided on a single process, and global ones, which depend on several processes. There is also a distinction between stable properties and unstable ones. We would like to introduce another kind of classification. As we already said, the observer may receive observable events in any order. We say that a consistent cut is appearing to the observer when all its maximal elements (for $\prec$ ) have been observed. This means that in the subposet defined by already observed events, we already have the antichain which defines the ideal of the complete poset corresponding to the consistent cut. We are interested here in determining when the observer can decide a property on appeared consistent cuts.

We will say that a property is wait-free (WF for short) if for any ordering in which the observer receives events, the property may be decided on a consistent cut as soon as it appears. For example, the property $a \| b$, which means the concurrency of two events labeled with $a$ and $b$, is $W F$. Since there is no assumption on the ordering of received events, we can state that a property is WF iff it can be decided by only considering the maximal events of a consistent cut. For example, the property $a \prec b$, which is true for an ideal if it contains 
a maximal element labeled $b$ and in the past of this event, an event labeled with $a$, is not WF, since at the instant of observation of $b$ the decision cannot be taken.

However, among non WF properties, some have nice behaviors which sometimes allow decision before reception by the observer of the complete consistent cut, that is they do not necessarily need the knowledge of the whole past. There are properties which, when reaching a certain value (true or false) on an uncomplete ideal, definitely conserves this value on this ideal. For example $a \prec b$ is definitively true in a consistent cut containing a maximal event labeled with $b$ as soon as an event $a$ belonging to the past of $b$ is observed. There is also properties which can be decided when a certain part of the cut is known. For example, $a \prec b$ (" $a$ is covered by $b$ ") can be decided as soon as all the immediate predecessors of $b$ in $\widetilde{O}$ are known.

Note that the membership of properties to the aforementioned classes can be modified when assumptions can be made on the possible orderings in which events are received by the observer.

There is a practical consequence to the above sketch of classification. If we choose a direct dependence coding, in the construction of the lattice we must wait for linear extensions, thus our classification is unaccurate. On the other hand, with the vector timestamp coding, we can always decide a WF property in an ideal as soon as it appears to the observer. This allows an early decision at the cost of a more expensive intrusion.

\section{Checking of regular properties}

In this section we are interested in the problem of verifying properties of traces during the construction of the ideal lattice. Model-checking is a well known verification technique which consists in deciding whether a logical temporal formula is satisfied by a program. Model-checking is based on the standard model of transition systems which are essentially labeled digraphs used to represent the set of possible behaviors of the program. Numerous works have been developed these ten last years to justify the interest of temporal logics (linear or branching time) in this context, and to design general model-checking algorithms based on graph traversals. We will show that the ideal lattice can be viewed as a labeled transition system. Therefore, no doubt that the aforementioned techniques can be applied to the particular graph of ideals. Though this question has not yet been studied to our knowledge, we expect a great simplification due to the algebraic structure of the graph. One interest here is to take into account the requirement of on-line computation [JJ89].

Surprisingly, the approach in distributed debugging was different. Only very restrictive classes of properties with respect to the general framework provided by temporal logics (see [Pnu86] for example) have been studied. Our feeling is that the ideas about detection algorithms have preceded the properties formalization (one specifies only what is known to be detectable). There is also the wish to limit the exploration of the set of possible behaviors to keep a reasonable algorithmic complexity.

Our ambition is to show that existing techniques can be adapted to this context. As a starting point, we want to describe some general algorithms, able to compute a large class 
of properties. These properties are specified by finite automata. Consequently one often uses the term automaton verification instead of model-checking. However, as every formula of linear time temporal logic (LTL) interpreted over finite sequences can be automatically translated into a finite automaton which accepts the same models, we already reach the power of LTL.

\subsection{Ideal lattice and transition system}

A (deterministic) labeled transition system is a triple $T=(Q, A, \longrightarrow)$ where $Q$ is the set of states, $A$ the alphabet of actions, and $\longrightarrow \subseteq Q \times A \times Q$ is the deterministic transition relation. The covering digraph of the ideal lattice $\widetilde{I(O)}$ of the poset $O$ can be viewed as a labeled transition system:

$$
\operatorname{Tr}(I(O))=<I(O), \Sigma, \longrightarrow>
$$

where the set of states is the set $I(O), \Sigma$ is the alphabet of actions (which occur as observable events), and $\longrightarrow$ is defined by $I \stackrel{a}{\longrightarrow} J$ iff $I-\underset{I(O)}{\widetilde{C}} J$ and $\lambda(J-I)=a$.

\subsection{Regular properties}

Let us now define the class of regular properties defined by finite automata on finite words. In each maximal path of the lattice, the successive encountered labels form a word on $\Sigma$. The set of such words is $\lambda(\operatorname{lin}(O))^{3}$ where $\operatorname{lin}(O)$ is the set of linear extensions of $O$.

A finite (deterministic) automaton is a 5 -uple $\phi=\left\langle\Sigma, Q, q_{0}, F, \delta>\right.$, where

- $\Sigma$ is the action alphabet,

- $Q$ is the set of states,

- $q_{0}$ is the initial state,

- $F \subseteq Q$ is the set of accepting states,

- $\delta \subseteq Q \times \Sigma \times Q$ is the transition function.

These automata allow to specify properties about the potential or necessary sequencing of actions.

Let $\mathcal{L}(\phi)=\left\{u \in \Sigma^{*}: \delta^{*}\left(u, q_{0}\right) \in F\right\}$ be the language defined by $\phi$. Confronting such an automaton with the covering digraph of the lattice requires to consider, for each ideal $I$, the set Path $(I)$ of all paths from bottom to $I$. This leads to two possibilities when defining the satisfaction of a property $\phi$ in an ideal $I$ :

$$
\begin{array}{lll}
I \models_{\forall} \phi & \text { iff } & \lambda(\operatorname{Path}(I)) \subseteq \mathcal{L}(\phi) \\
I=_{\exists} \phi & \text { iff } & \lambda(\operatorname{Path}(I)) \cap \mathcal{L}(\phi) \neq \emptyset
\end{array}
$$

\footnotetext{
${ }^{3}$ For simplicity, we denote by $\lambda\left(e_{1} \ldots e_{n}\right)$ the word $\lambda\left(e_{1}\right) \ldots \lambda\left(e_{1}\right)$.
} 
The satisfaction relation $\models_{\forall}\left(\right.$ resp. $\left.\models_{\exists}\right)$ states that an ideal $I$ satisfies $\phi$ iff every (resp. some) path in $\operatorname{Path}(I)$ is labeled with a sequence accepted by the automaton $\phi$.

Let $\neg \phi$ denote the complementary automaton of $\phi$. We have $\mathcal{L}(\neg \phi)=\Sigma^{*}-\mathcal{L}(\phi)$. Since for every $A, B \subseteq \Sigma^{*}$, we have $A \subseteq B$ iff $A \cap\left(\Sigma^{*}-B\right)=\emptyset$, and using the definitions of the satisfaction relations, we get the usual duality relation: $I \models_{\forall} \phi \Leftrightarrow I \forall_{\exists} \neg \phi$.

The upper element of $\widetilde{I(O)}$ is the ideal $O$ which contains all events of the trace. The satisfaction of $\phi$ by the lattice $I(O)$ is then defined by:

$$
\begin{array}{lll}
I(O) \models_{\forall} \phi & \text { iff } & O \models_{\forall} \phi \\
I(O) \models_{\exists} \phi & \text { iff } & O \models_{\exists} \phi
\end{array}
$$

From the above definitions, we can easily deduce that:

$$
\begin{array}{lll}
\forall I \in I(O), I \models_{\forall} \phi & \Leftrightarrow & \phi(I) \subseteq F \\
\forall I \in I(O), I \models_{\exists} \phi & \Leftrightarrow & \phi(I) \cap F \neq \emptyset
\end{array}
$$

where $\phi(I)=\delta^{*}\left(\lambda\left(\operatorname{lin}\left(O_{\mid I}\right)\right), q_{0}\right)$ is the set of states of the automaton $\phi$ reachable by paths ending in $I$. The checking algorithms will be automatically derived from these expressions of the satisfaction relations.

\subsection{The checking algorithms}

As was evoked in the preceding paragraph, the checking algorithms are essentially based on the computation of $\phi(I)$. It is easy to see that $\phi(I)$ can be computed on-line during the lattice construction since it is sufficient to attribute the ideals by states of the automaton $\phi$. In fact, the attribute $\phi(I)$ of an ideal $I$ can be computed from the attributes of its immediate predecessors in the lattice ${ }^{4}$ :

$$
\phi(I)=\bigcup_{J \in\rfloor_{I(0)}^{i m} I}\left(\bigcup_{q \in \phi(J)} \delta(\lambda(J-I), q)\right)
$$

The algorithm can be implemented by a breadth-first traversal of the lattice during which each ideal is attributed with a boolean array of size $|Q|$, the $i^{\text {th }}$ element indicating whether some sequence leading to $I$ can put the automaton in state $i$. If we want to extend the algorithm in order to count the number of sequences accepted by the automaton, it suffices to replace the boolean array by an array of integers.

It is straightforward to see that the complexity of the checking algorithms is linear in the size of the lattice and the size of the automaton.

Figure 5 illustrates the result of algorithms for checking $\models_{\forall}$ and $\models_{\exists}$. The subset of the lattice denoted $E$ represents the set of ideals $I$ such that $I \|_{\exists} \phi$ and the subset $A$ consists of ideals satisfying $I \models_{\forall} \phi$.

\footnotetext{
${ }^{4}$ This supposes that events are treated according to a linear extension of the poset.
} 


\section{References}

[BDM*94] P. Baldy, H. Dicky, R. Medina, M. Morvan, and JF. Vilarem. Efficient reconstruction of the causal relationship in distributed computations. In Canada-France Conference on Parallel Computing, Montréal, Canada, May 1994.

[Bir67] G. Birkhoff. Lattice theory. In American Mathematica Society Colloquium Publications, Vol. XXV, 1967.

[BP82] R. Bonnet and M. Pouzet. Linear extension of ordered sets. In I. Rival, editor, Ordered Sets, pages 125-170, D. Reidel Publishing Company, 1982.

[BR94] O. Babaoglu and M. Raynal. Specification and verification of behavioral patterns in distributed computations. In Proc. of the $4^{\text {th }}$ Int. Conference on Dependable Computing for Critical Applications, Springer Verlag Series, San Diego, January 1994.

[CL85] K. M. Chandy and L. Lamport. Distributed snapshots: determining global states of distributed systems. ACM TOCS, 3(1):63-75, 1985.

[CM91] R. Cooper and K. Marzullo. Consistent detection of global predicates. In Proc. ACM/ONR Workshop on Parallel and Distributed Debugging, pages 163-173, Santa Cruz, California, May 1991.

[DJR93] C. Diehl, C. Jard, and J.X. Rampon. Reachability analysis on distributed executions. In M.C. Gaudel and J.P. Jouannaud, editors, TAPSOFT, pages 629-643, Springer-Verlag, LNCS 668, Orsay, April 1993.

[Fid88] J. Fidge. Timestamps in message passing systems that preserve the partial ordering. In Proc. $11^{\text {th }}$ Australian Computer Science Conference, pages 55-66, February 1988.

[FZ90] J. Fowler and W. Zwaenepoel. Causal distributed breakpoints. In $10^{\text {th }}$ IEEE International Conference on Distributed Computing Systems, pages 134-141, 1990.

[GW92] V.K. Garg and B. Waldecker. Detection of unstable predicates in distributed programs. In Proc. of the 12th conf. Foundations of Software Technology and Theoretical Computer Science, pages 253-264, Lecture Notes in Computer Science 652, Springer-Verlag, New Delhi, India, December 1992.

[JJ89] C. Jard and T. Jéron. On-line model-checking for finite linear temporal logic specifications. In Proceedings of the International Workshop on Automatic Verification Methods for Finite State Systems, pages 275-285, Grenoble, France, June 1989. Springer-Verlag, LNCS 407.

[Lam78] L. Lamport. Time, clocks and the ordering of events in a distributed system. Communications of the ACM, 21(7):558-565, July 1978. 
[Mat89] F. Mattern. Virtual time and global states of distributed systems. In M. Cosnard, P. Quinton, M. Raynal, and Y. Robert, editors, Proc. Int. Workshop on Parallel and Distributed Algorithms Bonas, France, Oct. 1988, North Holland, 1989.

[Pnu86] A. Pnueli. Applications of temporal logic to the specification and verification of reactive systems: a survey of current trends. LNCS 224, Current Trends in Concurrency, 510-584, 1986. 
Unité de recherche INRIA Lorraine, Technôpole de Nancy-Brabois, Campus scientifique, 615 rue de Jardin Botanique, BP 101, 54600 VILLERS LÈS NANCY

Unité de recherche INRIA Rennes, IRISA, Campus universitaire de Beaulieu, 35042 RENNES Cedex

Unité de recherche INRIA Rhône-Alpes, 46 avenue Félix Viallet, 38031 GRENOBLE Cedex 1

Unité de recherche INRIA Rocquencourt, Domaine de Voluceau, Rocquencourt, BP 105, 78153 LE CHESNAY Cedex

Unité de recherche INRIA Sophia-Antipolis, 2004 route des Lucioles, BP 93, 06902 SOPHIA-ANTIPOLIS Cedex

Éditeur

INRIA, Domaine de Voluceau, Rocquencourt, BP 105, 78153 LE CHESNAY Cedex (France)

ISSN 0249-6399 